Para enlazar con este artículo / To link to this article:

http://dx.doi.org/10.14198/fem.2019.33.03

Para citar este artículo / To cite this article:

Del Hoyo Moreno, Idoia. «El estigma al servicio del poder. Dominación y resistencias desde la interseccionalidad». En Feminismo/s, 33 (junio 2019): 65-90. Dosier monográfico: Diálogos entre la democracia participativa y la interseccionalidad. Construyendo marcos para la justicia social, coords. Patricia Martínez-García y Jone Martínez-Palacios, DOI: 10.14198/fem.2019.33.03

\title{
EL ESTIGMA AL SERVICIO DEL PODER DOMINACIÓN Y RESISTENCIAS DESDE LA INTERSECCIONALIDAD ${ }^{1}$
}

\author{
STIGMA AT THE SERVICE OF POWER \\ DOMINATION AND RESISTANCES THROUGH \\ INTERSECTIONALITY
}

\author{
Idoia Del HOYO MORENO \\ Investigadora Predoctoral \\ Universidad del País Vasco (UPV/EHU) \\ idoia.delhoyo@ehu.eus \\ https://orcid.org/0000-0002-7223-2906
}

\section{Resumen}

El objetivo de este artículo es el de contribuir a la comprensión y a la progresiva desactivación de las diversas estructuras de opresión que sujetan, constituyen e influyen en las experiencias vividas de los agentes subalternos. Concretamente, posa la mirada sobre un fenómeno que ha sido, hasta ahora, poco estudiado en Ciencia Política: el estigma. Así, a partir de una breve revisión teórica de la literatura sobre la estigmatización, trata de ofrecer algunas claves que contribuyan a su reconceptualización y posterior análisis, explorando, especialmente, el potencial analítico de los marcos interseccionales propuestos por Patricia Hill Collins. De este modo, el artículo explica de qué forma la matriz de dominación y el marco de los dominios de poder propuestos por la autora nos permiten comprender, por un lado, el papel que juega el estigma

1. Quisiera agradecer a las coordinadoras de este monográfico el trabajo y el esfuerzo que han realizado, así como a Jone Martínez-Palacios por su acompañamiento y sus comentarios. Agradezco también a las/los informantes del artículo sus sugerencias. 
en la dinámica interseccional del poder y, por otro, ahondar en las resistencias que movilizan los agentes estigmatizados para enfrentarlo.

Palabras clave: estigma, interseccionalidad, dominación, resistencia, Patricia Hill Collins.

\begin{abstract}
This article aims to contribute to the comprehension and progressive deactivation of the multiple structures of oppression that hold, constitute and influence the lived experiences of subaltern agents. Particularly, it focuses on a social phenomenon that has been hardly studied in Political Science: social stigma. Through exploring the analytical potential of the intersectional frameworks proposed by Patricia Hill Collins, the article offers some key ideas to promote the reconceptualization and subsequent analysis of social stigmatization. Thus, along with understanding how stigma works within the intersectional dynamic of power, both the matrix of domination and the domains of power framework proposed by the author will let us explore the resistance strategies that stigmatized agents mobilize in order to revert stigmatization.
\end{abstract}

Keywords: stigma, intersectionality, domination, resistance, Patricia Hill Collins.

\title{
1. INTRODUCCIÓN
}

\section{Estigma}

(Del lat. Stigma 'marca hecha en la piel con un hierro candente', 'nota infamante')

1.m. Marca o señal en el cuerpo.

2.m. Desdoro, afrenta, mala fama.

3.m. Huella impresa sobrenaturalmente en el cuerpo de algunos santos extáticos, como símbolo de la participación de sus almas en la pasión de Cristo. 4.m. Marca impuesta con hierro candente, bien como pena infamante, bien como signo de esclavitud.

6.m. Med. Lesión orgánica o trastorno funcional que indica enfermedad constitucional y hereditaria.

El término estigma, que deriva del latín stigma, hace referencia, ya en sus acepciones más primitivas, a una marca, especialmente a aquellas que se grababan sobre el cuerpo de animales y personas con un hierro candente en la antigüedad clásica (Goffman 13; Jones 140). Pese a haber estado sujeto a diversas 
modificaciones -especialmente relevantes a partir de la Edad Moderna- su significado se mantiene todavía cercano al original, si bien ya no depende de la impresión corporal de la propia marca (Goffman 13). En consecuencia, las investigaciones más recientes sobre este fenómeno advierten su larga historia punitiva, que habría derivado de formas de estigmatización ejercidas a través de prácticas de violencia corporal, a otras más sutiles de violencia simbólica ${ }^{2}$ (Tyler, Hieroglyphics, 9-10). Muchos de los estudios, sin embargo, han fallado en destacar esta larga genealogía penal, de tal manera que «desde la segunda mitad del siglo XX nos hemos acostumbrado a pensar el estigma como un problema de normas sociales que puede ser enfrentado y aliviado a través de la comprensión y la educación» (Tyler, Hieroglyphics 9).

Es posible situar la conceptualización contemporánea del término a la que Tyler hace referencia a mediados de la década de los 60 , concretamente con la publicación de la obra Estigma: La identidad deteriorada del sociólogo canadiense Erving Goffman. Un trabajo que, incluso tras cinco décadas continúa siendo una importante «fuente de inspiración» (Pescosolido y Martin 88) para aquellas personas comprometidas con el estudio de la estigmatización social. Por ello, el marco conceptual de Goffman se sitúa en una posición hegemónica, de forma que se privilegian tanto su pensamiento como el enfoque micro-sociológico que empleó a lo largo de sus investigaciones. Ambas dinámicas han suscitado tantos adeptos como voces críticas, que apuntan a su aparente carácter «individualista, ahistórico y políticamente anestesiado» (Tyler, Stigma 764), en tanto que parte de una teorización que falla en considerar las relaciones de poder estructurales (Farrugia 1012) y desemboca, por tanto, en lecturas parciales sobre su impacto en las experiencias vividas de quienes son atravesados por él.

La tendencia a presentar la estigmatización como parte de una tipología más amplia de reacciones de rechazo, derivadas, de forma genérica, de los procesos de categorización social (Fiske 357), sugiere una comprensión que la sitúa como una respuesta individual y/o colectiva aparentemente naturalizada,

2. En este texto se emplean los términos estigma y estigmatización en su uso más contemporáneo. Esto supone que, si bien el estigma fue empleado como forma de castigo penal formal en la Antigüedad y en la Edad Media, aquí nos referimos a él a partir de su configuración más reciente.

Feminismo/s 33, junio 2019, pp. 65-90 
que nos guía, en última instancia, a la aceptación de las jerarquías sociales como parte de la formación identitaria de los sujetos.

Referimos, en este sentido, dos problemáticas generales en torno al estudio del estigma y la estigmatización que motivan, de manera inicial, la escritura de este artículo. Por un lado, identificamos una creciente preocupación en relación a los obstáculos teórico-analíticos provenientes de la literatura sobre el estigma; una literatura que ha desatendido en cierta medida la complejidad inherente a la estigmatización. Por otro, se atribuye a los agentes estigmatizados una cierta pasividad y victimización (Farrugia 1012); una quietud que bebe de una concepción concreta del poder como fuerza opresiva y que sugiere el carácter estático e irremediablemente permanente de la estigmatización social.

Así, en relación con la primera de las percepciones mencionadas, asistimos desde principios de siglo a la proliferación de investigaciones que, recuperando las teorizaciones mencionadas, han tratado de ampliar y completar las lecturas iniciales (Pescosolido y Martin 88-91). Este regreso del estigma al campo académico ha promovido premisas más relacionales y dinámicas, crecientemente preocupadas por subrayar la importancia y centralidad que adquiere el estigma en los procesos de producción y reproducción de una multiplicidad de fenómenos sociales y que van más allá del plano de las interacciones personales. Es el caso de complejas dinámicas como la ghettización y zonificación espacial urbana que fue denominada estigmatización territorial por Wacquant (67-69) o la expulsión y restricción en el acceso a servicios sociales de las poblaciones de mayor edad que Latimer ha estudiado recientemente (832-856).

Estos trabajos nos permiten pensar en torno a la posibilidad de que el estigma pueda ser algo más que una reacción cognitiva y/o afectiva. Nos permite cuestionarnos sobre la posibilidad de que este opere más allá del plano de las interacciones sociales, de forma que poseería una dinámica compleja y propia que, al tiempo que se sostiene sobre los sistemas de dominación existentes, los reproduce y los legitima (Tyler y Slater 727).

Pero del mismo modo que el estigma ha regresado a la esfera académica, diversos colectivos estigmatizados han reclamado también su lugar en la 
esfera pública ${ }^{3}$, lo que nos devuelve a la segunda de las percepciones arriba mencionadas. Éstos, que han sido denominados por la socióloga británica Imogen Tyler como «las voces de la resistencia», «contra-públicos dentro de los límites del Estado que, a partir de sus protestas fracturan y disputan las ideologías coercitivas, las injusticias y las desigualdades cada vez más profundas de una democracia degradada» (Tyler, Revolting 2), nos llevan a abandonar aquellas miradas que los presentaban como «víctimas desprovistas de poder» (Farrugia 1012) y que incurrían en la naturalización y normalización de la experiencia estigmática, para reivindicar su capacidad de agencia y pensar sus acciones en términos de resistencia.

Lejos de pretender negar la naturaleza opresiva y excluyente de la estigmatización, la pasividad que les era atribuida nos sugiere una teoría del poder que la sitúa como fuerza inherentemente opresiva (Foucault, Power/ Knowledge 91-92). Un énfasis que nos guía, finalmente, a un escenario impregnado de pesimismo e imposibilidad de cambio. Así, a pesar de los importantes avances que estos retornos del estigma han supuesto tanto en el plano académico como en el político, su actualidad demanda nuevos marcos que nos permitan comprender e interpretar «la economía política de la estigmatización social» (Parker y Aggleton 17).

Unos marcos que, defendemos, deben partir de una comprensión del poder que no puede encorsetarse en lecturas que lo reducen a un instrumento únicamente coercitivo y que nos permita explicar la centralidad que adquiere la resistencia en la dinámica del poder (Foucault, History 95). Una idea que sugieren también las teóricas de la interseccionalidad cuando al observar, a partir de sus propias experiencias, que un individuo puede ser opresor y oprimido al mismo tiempo, «atrae la atención sobre los aspectos productivos del poder, principalmente, los caminos por los cuales el ejercicio del poder también produce inevitablemente posibilidades de resistencia» (Severs, Celis $\&$ Erzeel 46-47). De este modo, la ambivalencia inherente a la naturaleza

3. Si bien el trabajo de Tyler está situado en Inglaterra, en el Estado español también asistimos a una proliferación de colectivos organizados que denuncian su estigmatización y reclaman una inclusión real. Es el caso del Sindicato Popular de Vendedores Ambulantes (Barcelona); la constitución de una sección sindical de prostitutas Unión Sindical de Trabajo Sexual dentro del sindicato IAC (Barcelona) o el recién constituido colectivo de personas psiquiatrizadas Zoroa (Bilbao).

Feminismo/s 33, junio 2019, pp. 65-90 
productiva del poder, no únicamente revela sus propias fronteras, sino que ofrece la posibilidad de transgredir dichos límites desde la otredad (Bhabha 92).

En este sentido, defendemos que los marcos y herramientas interseccionales nos proporcionan elementos y posiciones interesantes desde donde pensar el estigma. Dada la complejidad con la que se experimenta la estigmatización, no concebimos el estigma como un fenómeno aislado, sino interdependiente de los sistemas de dominación interseccionales. Por ello, exploraremos, concretamente, las potencialidades que nos ofrece Patricia Hill Collins a partir de sus propuestas: la matriz de dominación y el marco de los dominios de poder (Black 277-288; Difference 26-27; Collins y Bilge 7-13). Defendemos que a partir de ellos es posible abordar el estudio del estigma de forma holística, ya que nos invita, por un lado, a entender los procesos de producción y reproducción en los que está inserto y, por otro, cómo opera a través de cada uno de los dominios. Es nuestra voluntad que este marco nos guíe hacia una lectura integral de las funcionalidades que juega el estigma en la producción y el mantenimiento de las jerarquías sociales a través de la legitimación de la desigualdad y la dominación de ciertas poblaciones, al tiempo que ofrece a los agentes estigmatizados una serie de caminos en forma de estrategias de resistencia.

En tanto que contribuir a la inclusión y presencia de las poblaciones subordinadas pasa inevitablemente por comprender la complejidad de sus ausencias y exclusiones, este artículo pone el foco sobre el estigma y la estigmatización social con el objetivo de ofrecer una propuesta teórico-analítica que no sea ajena a la dinámica interseccional del poder y nos permita comprender el papel que este encarna en la misma, tanto en términos de dominación como de resistencia. Objetivos, ambos, que tienen como horizonte orientar nuestro conocimiento y nuestras prácticas hacia un proceso de democratización comprometido con la consecución de sociedades más justas e inclusivas.

El artículo, entonces, estará organizado de la siguiente manera: en un primer momento presentaremos una revisión de aquellas obras que han resultado determinantes en la teorización del estigma, con el objetivo de proponer una serie de claves para su re-conceptualización. En segundo lugar, introduciremos la interseccionalidad, prestando especial atención al marco

Feminismo/s 33, junio 2019, pp. 65-90 
de los dominios de poder propuesto por Patricia Hill Collins. Finalmente, exploraremos las potencialidades del marco de Collins para leer y analizar los procesos de producción y reproducción de la estigmatización social y su papel dentro de la dinámica interseccional del poder.

\section{HACIA UNA LECTURA DEL ESTIGMA COMPROMETIDA CON EL PODER}

\subsection{Consideraciones iniciales: un breve recorrido por la literatura sobre el estigma}

Como hemos anunciado, las reflexiones de Goffman sobre el estigma han sido objeto de cuestionamiento y sometidas, en la última década, a un proceso de reelaboración constante. Así, en las siguientes líneas trataremos de exponer su desarrollo y resultados con el objetivo de identificar con una concreción mayor los principales obstáculos y avances referidos en la sección anterior.

Inicialmente, es importante señalar que el trabajo de Erving Goffman parte de la vocación de comprender las interacciones sociales y las formas en las que los procesos de categorización intergrupal hacen de ellas situaciones incomodas para algunos de los participantes (Tyler, Stigma 748-749). En este contexto, el sociólogo expuso la premisa por la cual toda sociedad establece las herramientas necesarias para clasificar y posteriormente categorizar las diferencias humanas en relación a ciertos atributos que conforman el espectro de lo normal (14). Estos, que se estructuran como expectativas normativas, son incorporados, de forma que se establece el rango de atributos aceptables en función de las categorías previamente identificadas. Así, cuando una persona se presenta ante nosotros portando un atributo alejado del rango de expectativas normativas que le corresponde y que serían propias de la categoría que parece habitar, se produce una ruptura identitaria que desemboca en un proceso de descrédito y devaluación (14-15). Si bien la teorización de Goffman es más compleja de lo que estas líneas permiten exponer, diremos que, en síntesis, este define el estigma como «un atributo profundamente desacreditador» (15) que hace de quien lo posee, alguien «menos apetecible» (14) para la interacción social.

Partiendo de esta definición, Crocker, Major y Steele afirmarán que el estigma es una identidad social negativa o, más concretamente, que este 
supone poseer un atributo que implica un proceso de devaluación identitaria en un contexto particular (506). Dada la insistencia en situar la estigmatización como un proceso contextual, el estigma se presenta como una amenaza universal, que, como veremos posteriormente, está sujeto a ciertas dimensiones que hacen de las condiciones e impacto de la estigmatización un fenómeno variable. Una idea que, aunque resultó sugerente, no escapa del enfoque cognitivo que parece envolver la literatura sobre la estigmatización social. Por ello, nos encontramos frente a reflexiones tendentes a naturalizar los procesos de clasificación y categorización de las diferencias humanas, de tal forma que asumen su aparentemente automática metamorfosis en expectativas normativas, al tiempo que lo sitúan como un tipo de reacción humana ${ }^{4}$.

Es por ello por lo que las investigaciones posteriores no tardaron en ampliar estas propuestas a través de la incorporación de nuevos elementos, proporcionando un marco crecientemente comprometido con las dinámicas de poder. Bruce Link y Jo Phelan, entre otros, subrayaron que la categorización de las diferencias humanas es vinculada con toda una serie de características indeseables -en forma de estereotipos negativos- como fase previa y necesaria en el mantenimiento de la distinción nosotros/otros, que determina un acceso desigual al estatus social y al poder político-económico en tanto estadios que «coexisten en una situación de poder que autoriza el despliegue de los componentes del estigma» (Conceptualizing 367). Afirmarán, en este sentido que «el estigma es completamente dependiente del poder social, económico y político -necesita del poder para estigmatizar» (Conceptualizing 375).

La obra de Link y Phelan supuso un giro normativo, gracias al cual se incentivaron lecturas de carácter procesual y relacional sobre el estigma (Barón Vioque, Cascone y Martínez Valle 840), que indicarían su atribución a partir de un proceso de producción y significación diferencial de las especificidades humanas a través de la asignación estereotipada, en detrimento de los marcos previos que lo situaban como una propiedad del individuo

4. Estimamos importante señalar que esta dinámica de normalización de la estigmatización pivota y viene precedida, al menos en parte, por el interés que Goffman mostró, desde el inicio, para con el estudio de las relaciones de poder, así como su falta de voluntad a dirigir sus conocimientos hacia el cambio y la justicia social (Tyler, Stigma 748-750).

Feminismo/s 33, junio 2019, pp. 65-90 
estigmatizado (Goffman 16) ${ }^{5}$. Si bien esta propuesta complejiza el estudio de la estigmatización social, situándola como un proceso social necesariamente marcado por las desigualdades, existen ciertos elementos que nos llevan a pensar en la relación entre estereotipado, estigmatización y dominación desde una lógica causal que, a nuestro juicio, resulta problemática para comprender, entre otras cosas, el origen de la estigmatización.

Precisamente, en un intento por profundizar en la etiología del estigma, Stangor y Crandall advierten sobre la importancia que representa el miedo, como emoción aversiva, en el proceso de estigmatización social, de manera que la piensan como una herramienta defensiva ante una posible amenaza que ponga en peligro el correcto funcionamiento del mundo social (74); es decir, que ponga en peligro las creencias culturales dominantes. Desde una perspectiva funcionalista y a través de una comparativa entre las ciencias naturales y sociales, proclaman que, en tanto todo organismo vivo tiende a rechazar y alejarse de aquello que estima amenazante, la misma operación se desarrolla en las interacciones sociales (74). Así, un atributo pasa a constituirse estigma cuando, por sus características concretas, supone una amenaza simbólica o tangible para el correcto funcionamiento del mundo social, frente a los ojos de su interlocutor (74).

Las consecuencias negativas que se derivan de la estigmatización, sin embargo, parecen depender de una serie de variables que hacen de ésta una experiencia gradual y, en consecuencia, cuantificable. En un libro pionero publicado por Edward Jones y sus colegas, estos proponen que los estigmas y, por ende, sus efectos, varían en relación a seis dimensiones: visibilidad, intensidad, incomodidad, estética, origen y peligro (Panchakis et al. 452453 $)^{6}$. Esto supondría que, en función de las características concretas del estigma, el grado de estigmatización puede ser mayor o menor, lo que nos sitúa no únicamente en una lógica aditiva de la opresión, sino en una lógica

5. A pesar de que Goffman afirma que el estigma demanda un «lenguaje de relaciones y no de atributos» (14), hace referencia al estigma como algo que el individuo posee: «la persona que tiene un estigma no es totalmente humana» (16) o «un atributo que estigmatiza a un tipo de poseedor» (16).

6. La propuesta de Jones ha sido recogida y reforzada por diversos autores, entre los que se encuentran Crocker, Major y Steele.; Link y Phelan (Conceptualizing) y más recientemente Pachankis et al.

Feminismo/s 33, junio 2019, pp. 65-90 
cuantificable de la misma ${ }^{7}$. Esta propuesta indujo rápidamente a los investigadores a pensar, como hemos señalado anteriormente, que del mismo modo que la estigmatización podía variar en base a las características concretas de la propia marca, el contexto social en el que se producía la misma también generaría diferencias (Crocker, Major y Steele 506). Igualmente, la posición de quienes participan en la interacción obtuvo una importancia central -habitualmente en forma de variable cuantificable- de tal forma que el género, la clase, la etnia, la edad, el nivel educativo y la religión, entre otros, se establecieron como variables capaces de determinar (a) la predisposición de un individuo para estigmatizar a los demás y (b) el grado de dureza y aceptación social que tendrán las actitudes discriminatorias (Martin et al. 50-67).

A raíz de estas y otras consideraciones, identificamos una tendencia a presentarlos como sujetos pasivos, cuyas acciones aparecen orientadas hacia la consecución de niveles aceptables de bienestar psicológico ${ }^{8}$ frente a una realidad difícilmente irreversible ${ }^{9}$ (Farrugia 2012). Sin embargo, Goffman ya apuntó que los individuos estigmatizados movilizan ciertas respuestas para manejar las interacciones marcadas por el estigma, donde destacan la racionalización normativa de la marca, la transformación correctiva del Yo y la defensa a través de la construcción de un Yo superior (22-24). A pesar de estas estrategias de manejo individuales tan ampliamente estudiadas, Regina Arnold y Gaylene Becker nos recuerdan la potencialidad de la acción colectiva a través de la conformación de movimientos sociales de protesta (51-52). Encontramos en la obra de Becker y Arnold una de las vías por las cuales el estigma puede aparecer al servicio del poder de resistencia de los colectivos estigmatizados.

7. Ejemplo de ello resulta la investigación realizada por Panchakis y sus colegas donde realizan un estudio empírico con el objetivo de clasificar y comprender el impacto del estigma (en términos de distancia social, bienestar psicológico etc.) en relación a las seis dimensiones propuestas por Jones.

8. El bienestar psicosocial superpone dos unidades de análisis (micro y meso) que agruparían, de un lado, el bienestar psicológico entendido en términos de salud mental -niveles controlados de ansiedad y estrés- y medidas subjetivas de bienestar -autoestima, actitudes positivas- y de otro el bienestar social o colectivo que hace referencia al soporte y el capital social, balance de esfuerzo-recompensa, tasa de autonomía y seguridad/ conflictos grupales (Eiroa-Orosa 41).

9. Major y O'Brien, entre otras, identifican tres estrategias de manejo del estigma: culpar a la discriminación de su situación; alejarse (disengagement) e incrementar la identificación grupal con personas igualmente estigmatizadas (404-406)

Feminismo/s 33, junio 2019, pp. 65-90 
$\mathrm{Y}$ es que, vemos de qué manera la constitución identitaria de los sujetos a través de una categoría estigmatizada supone una vía hacia la construcción no únicamente de un otros estigmatizado, sino de su posible conversión en un nosotros como estrategia de reafirmación y pertenencia, dirigida hacia la reconstrucción y valoración identitarias como proceso de cambio social (52).

En este sentido, y focalizando las dos últimas ideas esgrimidas, queremos hacer hincapié en los peligros adscritos a una comprensión de la experiencia de la estigmatización como realidad cuantificable, que no hace sino ahondar en la jerarquización de las vidas. Y es que el grado de dureza que tendrán las actitudes discriminatorias y estigmatizantes, no varía dependiendo del género, la raza, la clase o la edad de quien es estigmatizado/quien estigmatiza, sino que estas, en tanto categorías de división social, no solo generan experiencias complejas y únicas de desigualdad, sino que configuran, además, «espacios de resistencia y empoderamiento» (Cruells 37). En este sentido, cabe destacar que tanto la desigualdad como la organización del poder están formadas por una multiplicidad de ejes que se influyen mutuamente (Collins, Difference 20).

\subsection{Entre el castigo y el control social: el estigma como mecanismo de poder}

Como hemos visto, la teorización sobre el estigma ha venido preocupándose en demasía por las interacciones sociales, las bases psicosociales de la estigmatización y el impacto de la misma en el bienestar psicosocial, en detrimento del estudio de los elementos estructurales que hacen de este un mecanismo profundamente imbricado en los sistemas interseccionales de dominación (Parker y Aggleton 14-17). A este respecto, decíamos al inicio que el estigma ha regresado en la última década a la esfera pública. Un retorno que ha supuesto la proliferación de los trabajos, así como una mayor complejidad en las formas de abordar su estudio, fijando la mirada sobre problemáticas cada vez más amplias y numerosas. Así, encontramos trabajos que abordan las temáticas más tradicionales, como las enfermedades mentales, pero también otros que tratan cuestiones financieras, reproductivas, incluso el estigma relacionado al acento (Pescosolido y Martin 89). En la misma línea, cada vez son más también las publicaciones que tienen por objeto comprender este 
fenómeno a partir de sus implicaciones concretas en diversos países y/o instituciones, dando lugar a estudios comparados (89). No obstante, incluso las investigaciones más concretas ponen de manifiesto la necesidad de construir marcos más amplios que no sean ajenos a la complejidad que este enmascara.

Cuatro décadas antes de que Stangor y Crandall sugirieran que el estigma toma la función de herramienta defensiva ante la percepción de amenaza, la antropóloga británica Mary Douglas afirmaba en una investigación sobre el sentimiento de asco, la percepción de contaminación y los tabúes ${ }^{10}$ que «las reacciones de asco están siempre ancladas a creencias sociales más amplias» (Douglas, en Tyler, Revolting 23):

[Douglas] considera que el asco sirve para afirmar las fronteras del cuerpo social (el cuerpo político) a través de la expulsión (real o simbólica) de aquellos objetos, prácticas y personas que han sido colectivamente aceptadas como contaminantes. Así, la concienciación sobre la suciedad [...] revela las normas sociales que operan en un contexto social o cultural. (Meagher 32, en Tyler, Revolting 23)

De acuerdo con las palabras de Tyler sobre la investigación de Douglas, «las reacciones de asco son siempre contingentes y relacionales, de modo que revelan más información sobre la cultura en la que se experimenta y performa ese asco, que sobre el individuo u objeto que se estima asqueroso» (Tyler, Revolting 23). En este sentido, el asco, el miedo, la percepción de amenaza y otros sentimientos aversivos que guían a los individuos hacia el rechazo y la expulsión de los objetos y/o sujetos que los generan, responden a un proceso histórico de formación consensuada de la aversión, que es reproducido a través de su aprendizaje y consecuente transmisión. Las emociones aversivas resultan, entonces, profundamente políticas, ya que sirven como piedra angular para la producción y reproducción de las fronteras entre el nosotros y el otros (Ngai 338-339, en Tyler, Revolting 25). El estigma, entonces, en tanto herramienta defensiva frente a la percepción de amenaza (Stangor y Crandall 74), «no se corresponde con aquello que causa daño directamente [...] conductas que

10. Cabe señalar, a modo de contextualización, que Mary Douglas condujo su estudio a partir del análisis del concepto de pureza expuesto en diversas religiones y culturas primitivas. Destaca su estudio del Levítico XI donde se explica este concepto, especialmente a través de las prohibiciones alimenticias dispuestas en el judaísmo como impuras y, por tanto, contaminantes.

Feminismo/s 33, junio 2019, pp. 65-90 
están tipificadas como delitos»-que serían las que como sugieren los autores, amenazan el bienestar general-, sino que «se corresponde con lo que daña el orden de convivencia, lo que cuestiona los roles establecidos» (Juliano 22). Por ello, defendemos que la estigmatización vendrá precedida no únicamente por las creencias culturales dominantes en un contexto particular dado, sino por la producción normativa de los atributos y comportamientos potencialmente peligrosos para el mantenimiento del orden normativo dominante, que serán creados, transmitidos y aprendidos tanto por los individuos como por el entramado social e institucional (Becker y Arnold 46). Así, los estigmas son la respuesta ante las «faltas contra la jerarquía social» (Juliano 22).

El proceso de producción y reproducción de lo aversivo, entonces, adquiere la función de constituir y mantener las fronteras entre el nosotros y el otros. A raíz de la clasificación binaria característica del pensamiento occidental moderno que vino reforzada por las ciencias positivistas, la construcción identitaria de los individuos y los grupos se erige no únicamente sobre la dicotomía de un nosotros y un otros, sino, más específicamente, sobre la diferenciación de un nosotros dominante y un otros subalterno (Collins, Black 70) Como bien refiere la autora, la especificidad del pensamiento binario no radica únicamente en la categorización de los sujetos y los objetos en base a su diferencia, sino a aquella delimitada a partir de una relación de oposición (Black 70). Esta oposición constitutiva de la diferencia depende, como apuntó el pensador francés Michel Foucault, de la puesta en marcha de discursos de la verdad como escalones en la producción de la norma y, por ende, de la anormalidad (Vigilar 165-171).

Así, la producción de lo aversivo como mecanismo para el mantenimiento de la frontera identitaria nosotros/otros apoyada en el estigma, depende de la implementación de discursos que legitimen y justifiquen esa peligrosidad atribuida a determinadas características. Destacamos, como ejemplo, la construcción de las personas con menos recursos como peligrosas, que contribuye a la justificación de la austeridad en términos de recortes en derechos y recursos (Tyler and Slater, Stigma 727).

Vemos, de este modo, cómo tanto el miedo como el asco implican la existencia de una característica amenazante que denota la peligrosidad de quien 
inspira tales emociones, por lo que el concepto de peligrosidad ${ }^{11}$ adquiere, en el proceso de creación y atribución del estigma, un papel importante tanto para la producción de la norma como para la definición de sus parámetros de desviación (Juliano 18-23). Y es por ello también por lo que el estigma y la estigmatización social no pueden reducirse al dominio de las interacciones sociales, al nivel micro. En este sentido, siguiendo a Phelan, Link y Dovidio, el estigma posee -al menos- tres funciones, sensibles a constituirse todas ellas como riesgos o amenazas; en definitiva, el estigma actúa ante el peligro de (A) pérdida de poder -a través de la explotación; (B) infección -a través de la expulsión; (C) desorden social -a través de la restauración o el mantenimiento del orden normativo, en tanto herramienta para normalizar las poblaciones desviadas y mecanismo de advertencia para quien habita los límites de lo normativo (362).

Estas ideas nos invitan a leer el estigma como una herramienta para la explotación, expulsión y/o normalización de aquellas poblaciones estimadas peligrosas. Así, resulta en el castigo a la desviación; un mecanismo punitivo al servicio del control social y del mantenimiento de las fronteras normativas de comportamiento implantadas por el nosotros dominante. En la actualidad, y como comentábamos al principio, lo más habitual en relación a los castigos a la desviación, es que éstos se desarrollen a través de controles informales y que no se materialicen por los canales formales de penalidad contemporánea como los códigos penales o la encarcelación (Juliano 18), de manera que, como herramienta de castigo, el estigma ha sufrido esta metamorfosis de las formas punitivas generales hasta convertirse en una forma de violencia simbólica que pasa, inevitablemente, por componentes emocionales (Tyler, Hieroglyphics 9).

Pero a pesar de que este resulte de la producción consensuada de las normas sociales de comportamiento establecidas por el nosotros dominante frente al otros subalterno, no debemos perder de vista el dinamismo propio

11. Entendemos aquí peligrosidad como atribución necesaria para establecer el salto de la patologización de ciertos comportamientos a su percepción como criminalidad. Se trata, por tanto, de un proceso histórico amplio a partir del cual la menor de las infracciones se establecía como un peligro para la sociedad en su conjunto, con el objetivo de disciplinar los cuerpos; un proceso que bebió, entre otras cosas, de los discursos promovidos por el degeneracionismo y la criminología positivista (Campos 8)

Feminismo/s 33, junio 2019, pp. 65-90 
de la opresión y el privilegio (Collins, Black 246). Dado que la dominación se estructura a través del cruce o la intersección de diversos y múltiples sistemas de poder (Collins, Difference) el orden de lo estigmatizable variará en relación a la especificidad de dicho cruce, creando experiencias múltiples de dominación y lugares comunes para la resistencia.

\section{ESTIGMATIZACIÓN E INTERSECCIONALIDAD}

\subsection{Apuntes introductorios: sobre la propuesta de Patricia Hill Collins}

Como ya hemos expresado anteriormente, la propuesta central que vertebra este artículo incide en la idea de que tanto el estigma como la estigmatización social se leen mejor desde y a través de la interseccionalidad. Una apuesta de la que emana, además, un esfuerzo por construir un marco teórico-analítico que nos dé la posibilidad de estudiar el estigma más allá del plano micro e individual, así como despejar algunas de las incógnitas que caracterizan la complejidad de la estigmatización.

Si bien debemos situar el «nacimiento» de la interseccionalidad, como término, con la obra de Kimberlé Crenshaw, este no resultó sino un significante con el que nombrar y, así, traer al plano público la complejidad de la experiencia opresiva que enfrentaban muchas feministas, concretamente las mujeres negras (Collins y Bilge 65). Estas encarnan una experiencia de dominación que no puede ser comprendida desde marcos que piensan la subordinación a partir de una única categoría de división social; de manera que tampoco puede ser enfrentada ni resuelta desde esa misma posición (Crenshaw 140). De este modo, el paradigma interseccional nos da la oportunidad de pensar en la desigualdad y la injusticia a partir de la relación dependiente de múltiples sistemas de opresión (Collins, Black 18), principalmente porque la interseccionalidad «es una forma de comprender y analizar la complejidad en el mundo, en las personas y en las experiencias humanas» (Collins y Bilge 2). Patricia Hill Collins y Sirma Bilge nos invitan, además, a hacer uso de ella como un aparato heurístico, lo que la convierte en una herramienta «contraria a la simplificación en la comprensión de la opresión y la exclusión» (Martínez-Palacios 59-60). La «parcialidad»-comprendida en este caso como carencia de compleción- de algunas de las teorizaciones y análisis sobre el 
estigma, entonces, ha sido el detonante para apostar, finalmente, por desarrollar un marco desde donde acceder a la misma.

Siendo múltiples y diversos los caminos para acercarse a la interseccionalidad y siendo conscientes también de los riesgos que la acompañan (Martínez-Palacios 64), sugerimos un punto de entrada que pasa por dos marcos propuestos por la socióloga Patricia Hill Collins: la matriz de dominación y los dominios de poder. Esta apuesta se debe, primero, a que la propuesta de la autora está caracterizada por la flexibilidad, de manera que nos ofrece marcos sensibles de ser utilizados para estudiar y comprender una multiplicidad de realidades y experiencias (Collins y Bilge 4). A ello se le suma una propuesta que nace desde un firme compromiso con la justicia social, que adquiere su forma no únicamente a través de la teoría, sino también de la práctica política (Collins y Bilge 42; Collins, Difference 21).

Al respecto de la matriz de dominación la autora escribe: «El término matriz de dominación describe la organización social general dentro de la cual se origina, se desarrolla y se contiene la intersección de las opresiones» y añade que ésta "puede ser vista como una organización del poder históricamente específica, en la que los grupos sociales están insertos y en la que tratan de influir» (Collins, Black 228). En este sentido y como ella misma indica a modo de ejemplo, la matriz de dominación de las mujeres negras está constituida por la intersección de los ejes de opresión de raza, clase, género y sexualidad. Así, la figura de la matriz nos permite nombrar y organizar las complejas experiencias de dominación experimentadas por los sujetos.

Y si la matriz de dominación estructura los diversos ejes de división social que constituyen la experiencia de los sujetos y los grupos, esta está estructurada y opera a través de cuatro dominios de poder interrelacionados, sirviendo cada uno de ellos a una función u objetivo particular. Así, (A) el dominio estructural organiza la opresión, de manera que hace referencia a la organización de las instituciones sociales y a cómo estas estarían dirigidas a la reproducción/enfrentamiento de la subordinación; (B) el disciplinario la gestiona a través de la puesta en marcha y la utilización de las normas y las regulaciones de la vida cotidiana para mantener/enfrentar la jerarquía social; (C) el hegemónico o cultural justifica la opresión a través de la promoción de discursos e imágenes dirigidas, de nuevo, al mantenimiento/enfrentamiento de la injusticia; y, finalmente $(C)$ el dominio interpersonal influencia 
la experiencia cotidiana y la conciencia individual (Black 273-289; Difference 26; Collins y Bilge 7-13).

En este punto, vemos con especial claridad que la propuesta de la autora está ampliamente comprometida con la asunción de la capacidad de agencia y resistencia de las poblaciones subordinadas, de manera que nos sitúa de nuevo en una de las ideas que resaltábamos al inicio de este trabajo: que «allí donde hay poder, hay también resistencia» (Foucault, History 95). Así, subraya que, si bien las normas y regulaciones disciplinarias insertas en la vida cotidiana pueden ser empleadas como mecanismos para la reproducción de la desigualdad y la exclusión social, son igualmente susceptibles de ser utilizadas con el objetivo de fracturar las jerarquías sociales. Y es que «el argumento de los dominios de poder presentado aquí deberá servir para estimular diálogos sobre el empoderamiento» (Black 276).

\subsection{Leer el estigma desde la interseccionalidad: dominación y resistencia}

El psicólogo estadounidense Gregory Herek acuñó el término estigma sexual para denominar la estigmatización relativa a la desviación respecto de la norma de la heterosexualidad obligatoria (Barón, Cascone y Martínez 842), definiéndola como «el conocimiento compartido de la consideración social negativa hacia cualquier comportamiento, identidad, relación o comunidad no-heterosexual» (Herek 15). Con el objetivo de «incluir todos los procesos de estigmatización que tienen su origen en el sistema ideológico heteronormativo», Barón, Cascone y Martínez (844) propusieron ampliar la propuesta de Herek por el término estigma de género.

El trabajo de estas autoras nos invita a pensar el estigma en relación a cada uno de los ejes de división social, desde el género o la sexualidad, a la raza, la clase social o la religión; de manera que podríamos nombrar la consideración negativa respecto de las personas no-blancas estigma racial y respecto de las neurodivergentes estigma mental. Una idea que también fue sugerida por Becker y Arnold cuando se preguntaban por qué algunas mujeres que se desvían de las normas sociales son marcadas como criminales mientras otras no lo son (46-47). Esto nos sugiere que los estigmas, en tanto mecanismos punitivos y de control social, actuarían en relación a estructuras de dominación 
concretas que, como ya hemos señalado repetidamente, intersectan entre ellas constituyendo formas específicas de dominación política. Esta idea nos traslada a la matriz de dominación propuesta por Collins, ya que nos permite ordenar la función y el impacto del estigma en la dinámica interseccional del poder; un análisis que rara vez ha sido implementado respecto de este fenómeno. Así, el estigma no se limita únicamente al plano micro, sino que refiere una estructura de poder en forma de mecanismo dispuesto hacia el castigo y el control, interrelacionado con los diversos sistemas de opresión, permitiéndonos pensar acerca del lugar que ocupan estigma y estigmatización en la complejidad característica de la dominación.

Debemos recordar, con ello, que el poder está socialmente organizado a través de diversas dimensiones o dominios. De este modo, el marco dispuesto nos permite cuestionarnos sobre las formas en las que dichas relaciones de poder se distribuyen y se organizan, así como identificar y/o sugerir ciertas formas de resistencia dentro de cada matriz. Pero independientemente de la matriz que constituya la experiencia de una población estigmatizada concreta -que deberá ser desvelada desde del conocimiento de la experiencia vivida de cada agente y/o población a partir de un análisis empírico-, el marco de los dominios es susceptible de ser utilizado para estudiar los sistemas de poder tanto de forma conjunta como individual, lo que nos permite afinar la mirada en relación a la organización concreta de un cierto eje dentro de la dinámica interseccional del poder (Difference 27).

De este modo, proponer una lectura interseccional del estigma empleando el marco de los dominios de poder, implica cuestionarnos sobre los discursos, las prácticas y las instituciones a través de las cuales se organiza (dominio estructural), se gestiona (disciplinario), se justifica (hegemónico/cultural) y es subjetivada (interpersonal) la estigmatización social en un contexto espacio-temporal concreto. Pero del mismo modo, es también cuestionarnos acerca de las formas que adopta la resistencia a través de los dominios porque, como bien resalta la autora, cada una de estas prácticas guía a los agentes hacia formas de resistencia específicas de cada dominio, así como hacia un activismo político que sería transversal a los mismos (Collins Difference 27).

En este punto, y a propósito del dominio hegemónico, conviene subrayar que, tal y como afirma la autora, este involucra un «sistema de sentido común» (re)producido por los grupos dominantes (Black 284). Es por ello 
por lo que no solo debemos estudiar las imágenes y los discursos que lo componen, sino especialmente el complejo proceso a través del cual estos se configuran como parte de los sistemas de creencias compartidas.

Así, y en tanto la constitución de un cierto atributo y/o comportamiento como estigma depende de un proceso de producción social e histórico de estos como negativos o más concretamente, como peligrosos frente al mantenimiento del orden normativo dominante, el análisis del estigma pasa por comprender, por un lado, la producción y reproducción de lo aversivo y, por otro, su organización social. Esto implica, primero, identificar tanto las prácticas como los discursos que han sido empleados para construir ciertas características como peligrosas y, por tanto, indeseables, y que constituyen el sustrato necesario para justificar la expulsión de determinadas poblaciones; segundo, cómo han sido legitimadas e institucionalizadas; y tercero, conocer bajo qué condiciones y de qué manera dirige el sujeto sus prácticas hacia la reproducción/enfrentamiento de su subordinación.

La estigmatización entendida como necesidad de lejanía y, por tanto, rechazo hacia aquellas personas que portan un atributo que los convierte en personas «menos apetecibles» para la interacción social (Goffman 14), hace referencia, de este modo, a la experiencia cotidiana de la estigmatización o, dicho de otro modo, al dominio interpersonal de la misma. El hecho de que el estigma resulte un fenómeno colectivo que emerge en las interacciones cotidianas, nos indica que se trata de una estructura de subordinación histórica que ha sido significada y aprehendida tanto a nivel individual como colectivo. Sin embargo, y como subrayábamos en la sección anterior, en tanto fenómeno social, este no responde al orden de lo natural, sino al orden de lo normal. $\mathrm{La}$ (re)producción de lo estigmatizable, entonces, supone la implementación de una serie de discursos y prácticas, así como de la puesta en marcha de diversos mecanismos que, por un lado, justifiquen su naturaleza aversiva, es decir, del dominio hegemónico y, por otro, gestionen y legitimen su funcionamiento, en este caso, a partir del dominio disciplinario y del estructural. Así, independientemente de la matriz que constituya la posición de un sujeto y/o población, proponemos que es posible trazar un análisis del estigma a través del marco propuesto.

Si bien el recorrido que hemos iniciado por la literatura sobre la estigmatización nos da muchas pistas e ideas clave de cómo esta opera en las 
interacciones sociales y también de cómo se estructura e institucionaliza y los mecanismos que aúna, creemos que tanto la teorización del estigma como su posterior análisis no pueden ignorar la relación que existe entre este fenómeno y los ejes de opresión interseccionales. Ya sea nuestro objetivo estudiar la estigmatización que recae sobre las personas transexuales, las que ejercen la prostitución o aquellas neurodivergentes, una lectura del estigma desde la interseccionalidad implica cuestionarnos lo siguiente: ¿qué discursos, prácticas e instituciones interfieren en la construcción de ciertas poblaciones como aversivas?

Tomando el estigma asociado a la prostitución como ejemplo, la rigidez de las normas de comportamiento asignadas a cada género supone la construcción de un modelo único de ser mujer, de forma que muchas conductas, atributos y características de las mujeres son consideradas desviadas (Juliano 22-23). Así, a la no-participación de los roles designados al sexo femenino, le es asignada una valoración negativa como mecanismo punitivo sobre el que se erige la definición discursiva de la feminidad normativa. Vemos de este modo cómo aquellas mujeres que se han alejado del modelo único de feminidad tradicional -enraizado sobre la figura de la madre- han sido históricamente tratadas como desviadas, si bien esta concepción ha tenido configuraciones dispares (Juliano 22-23). La estigmatización de estas mujeres, entonces, pivota sobre una serie de discursos e imágenes que legitiman y justifican su peligrosidad, (hegemónico/cultural), alimenta un sistema formal de sanción penal que depende, directamente, de la sanción informal implementada a través del estigma (disciplinario) y es organizada y regulada a través de, por ejemplo, Leyes de Extranjería que refuerzan los controles migratorios e impiden la entrada de personas migradas y refugiadas bajo la máxima de «combatir el tráfico de personas con fines de explotación sexual» (estructural).

En el caso de las mujeres que se consideran sospechosas de ser víctimas de trata, comprobamos cómo el estigma opera realmente tanto en la legislación como en la actuación de los poderes públicos, es decir, en el dominio estructural y que consta de toda una red de vigilancia y disciplinamiento. Un informe realizado por Women's Link Worldwide en el año 2017 mostraba, al respecto de los hijos e hijas de estas mujeres que:

La mirada estereotipada sobre la forma en la que las mujeres nigerianas ejercen el maternaje y el cuidado de sus hijos e hijas, unido al control de 
la red, hace que las decisiones de las administraciones de interferir en su vida privada y familiar se activen más rápidamente. El estigma asociado a los contextos de prostitución donde sufren la explotación, atravesados por situaciones de exclusión social, provoca que las administraciones duden de que la educación y el entorno que pueden proporcionar estas madres a sus hijos e hijas sea la correcta. Estos factores contribuyen a un aumento de las decisiones de retirar la guardia y custodia de los niños y niñas que, en ocasiones, vulneran el derecho a la vida privada y familiar de estas mujeres y sus hijos. Para ellas, el mensaje que envía la administración no es un mensaje de protección, sino de criminalización y castigo. (24)

Vemos de este modo que la estigmatización de las mujeres víctimas de trata y/o sospechosas de serlo, no solo es organizada a partir de los cuatro dominios, de manera que somos conscientes de la complejidad que este encierra, sino que el estigma intersecta con otros sistemas de opresión; en este caso con la etnia y la clase. El marco de Collins nos muestra, así, la economía política de la estigmatización (Parker y Aggleton 17; Tyler y Slater 729).

Pero la autora nos invita también a pensar en términos de resistencia; es decir, en las posibles prácticas subversivas que los agentes movilizan en cada uno de los dominios, en tanto «esos mismos dominios han sido y son susceptibles de ser utilizados como espacios para el empoderamiento» (Black 277). Así, señala que los discursos contra-hegemónicos y la elaboración de conocimientos subyugados (hegemónico); el uso estratégico de regulaciones normativas (disciplinario); las luchas a favor de reformas legislativas (estructural) y las prácticas cotidianas empleadas como pequeñas formas de supervivencia (interpersonal), conforman todas ellas formas de resistencia a la opresión (Black, 277-288). Con ello, identifica también formas de resistencia que serían transversales a los cuatro dominios, más concretamente a partir de la organización y el activismo político (Difference 27). Esto sugiere que, como planteaban Becker y Arnold, el otros estigmatizado es susceptible de devenir en un nosotros (52). Aquí reside, precisamente, la idea más sugerente de este marco y es que, nos invita a cuestionarnos sobre la posibilidad de que aquello que construye a los sujetos y constituye su exclusión, sea precisamente aquello que permita e impulse sus alianzas y estrategias de resistencia. 


\section{CONCLUSIONES}

Dábamos comienzo a esta reflexión diciendo que la inclusión de las personas y colectivos estigmatizados pasaba, entre otras cosas, por comprender cómo se estructura y opera su exclusión. Así, a lo largo de estas páginas hemos tratado de reflexionar sobre cómo se ha constituido dicha exclusión, acercándonos, concretamente, a la dinámica de la estigmatización social. Era nuestro objetivo ofrecer un lugar desde donde mirar y explicar la economía política del estigma, de manera que comprendiésemos el papel que éste ocupa en el mantenimiento de las jerarquías sociales y la legitimación de la desigualdad. En este sentido, hemos tratado de mostrar las potencialidades de leerlo desde los marcos que nos ofrece Patricia Hill Collins.

Decía Iris Marion Young que, si bien el aparente compromiso que ha surgido con la igualdad ha dado pie a pensar que «la objetivación y evidente dominación de los cuerpos depreciados que imperaba en el siglo XIX ha retrocedido», lo cierto es que estas prácticas «han sido soterradas al tiempo que perviven en los hábitos cotidianos y en los significados culturales respecto de los cuales la gente, por lo general, no es consciente» (Young 210). Pero a pesar del componente emocional de la estigmatización, y también de su cotidianeidad, creemos que el marco presentado nos muestra que este depende de toda una serie de imágenes, discursos y creencias negativas que justifican y legitiman el rechazo y que, lejos de ser una respuesta cognitiva inconsciente, se encuentra institucionalizado, de forma que está muy presente tanto en la legislación como en las políticas públicas.

Somos conscientes de que la dominación de las personas y colectivos estigmatizados es más compleja de lo que hemos podido avanzar a partir de este ejemplo y de que esta no se reduce al estigma. También de que tanto el uso de la matriz de dominación, como del marco de los dominios de poder, se traducen en acceder a ellos desde la experiencia de vida de las mujeres (Martínez-Palacios 65). Sin embargo, y recuperando de nuevo las palabras de Young:

Si la opresión contemporánea se gesta a través de una estética del cuerpo, a través del nerviosismo y el rechazo motivados por las amenazas al sistema básico de seguridad y a través de imágenes y estereotipos que simultáneamente alimentan tal conducta, la legitiman y disipan los temores que ella 
expresa, entonces la reflexión normativa de la justicia debería incluir tales fenómenos. (Young 251).

Es por ello por lo que consideramos que la propuesta de pensar el estigma y la estigmatización social desde la interseccionalidad, no solo nos ofrece un espacio desde donde reflexionar y abogar por la justicia social, a través de la ruptura de los procesos de producción y reproducción del estigma, sino también la posibilidad de transformación y cambio social radical, en tanto que la complejidad con la que se configura y experimenta la estigmatización, así como el reconocimiento y asunción de la capacidad de agencia por la que aboga, nos muestran que «la relación dialéctica entre opresión y activismo es mucho más compleja» de lo que sugieren quienes entienden el poder en términos exclusivamente opresivos (Black 289). Y es que, si enfoques y conceptualizaciones previas como las de Goffman, hablaban del manejo de las interacciones marcadas por la estigmatización social a través de, por ejemplo, la adopción de un Yo superior (22-24), la lectura del estigma a partir de la interseccionalidad nos ofrece la posibilidad de preguntarnos si estas estrategias de manejo no constituyen también espacios para el empoderamiento individual y colectivo; si no constituyen lugares desde donde, como decía Tyler (Revolting 2), disputar aquellos discursos, prácticas e instituciones que justifican, legitiman y organizan la compleja arquitectura de la estigmatización y que nos guíen hacía un proceso de transformación social radical.

Así, el final de este artículo nos sitúa frente al que probablemente sea el objetivo más complejo de todos; precisamente el de dirigir nuestro conocimiento y praxis política hacia la desactivación de la estigmatización social desde un compromiso firme con la consecución de sociedades más justas, inclusivas y, por ende, democráticas. En ese camino, consideramos que aprender de las estrategias de resistencia que estos colectivos emplean para enfrentar su subordinación, constituye la forma más respetuosa de desactivar la estigmatización. Y es que estos ya están reclamando un espacio en la esfera pública, no solo a partir de la organización y el activismo político sino también desde el plano institucional. Es nuestra tarea, entonces, cuestionar los marcos existentes y proponer ideas para una reconceptualización crítica; pero lo es, especialmente, partir de premisas epistemológicas que impliquen «la necesidad de escuchar y tratar de entender las voces que proceden de diversos sectores sociales, y oponen ciertas barreras a los prejuicios» de manera que 
podamos contribuir a «desenmascarar algunas de las falacias legitimadoras más palpables» (Juliano 12).

\section{BIBLIOGRAFÍA}

Arnold, Regina y Gaylene Becker. «Stigma as a social and cultural construct». The Dilemma of Difference. Eds. Stephen C. Ainlay, Gaylene Becker y Lerita M. Coleman. New York, 1986. 39-57.

Barón Vioque, Susana, Michele Cascone y Carlos Martinez Valle. «Estigma del sistema de género: aprendizaje de los modelos normativos, bullying y estrategias de resiliencia». Politica y Sociedad 50.3 (2013): 837-864.

Bhabha, Homi K. «La otra pregunta. El estereotipo, la discriminación y el discurso del colonialismo». El lugar de la cultura, Buenos Aires: Ediciones Manantial, 2002.

Collins, Patricia Hill y Sirma Bilge. Intersectionality. Cambridge: Polity Press, 2016.

Collins, Patricia Hill. Black Feminist Though. Knowledge, Consciousness and the Politics of Empowerment. New York: Routledge, 2000.

- . "The difference that power makes: intersectionality and participatory democracy». Investigaciones Feministas 8.1 (2017): 19-39.

Crenshaw, Kimberlé. «Demarginalizing the Intersection of Race and Sex: A Black Feminist Critique of Antidiscrimination Doctrine». University of Chicago Legal Forum 1 (1989): 139-167.

Crocker, Jennifer, Brenda Major y Claude Steele. «Social Stigma». The handbook of social psychology. Eds. Daniel T. Gilbert, Susan T. Fiske y Gardner Lindzey. 2. New York, 1998. 504-554.

Cruells, Marta. La interseccionalidad politica: tipos, factores de entrada en la agenda política, jurídica y de los movimientos sociales. Barcelona: Universidad Autónoma de Barcelona, 2015.

Eiroa Orosa, Francisco Javier. «Cambio sociocultural y bienestar psicosocial. Una propuesta para la investigación y la acción». Psicología Política 43 (2013): 39-53.

Farrugia, David. «Exploring stigma: medical knowledge and the stigmatization of parents of children diagnosed with autism spectrum disorder». Sociology of Health E Illness 31.7 (2009): 1011-1027. 
Fiske, Susan T. «Stereotyping, prejudice and discrimination». The Handbook of Social Psychology. Eds. Daniel T. Gilbert, Susan T. Fiske y Gardner Lindzey. 2. New York, 1998. 357-411.

Foucault, Michel. History of sexuality, Vol.1. New York: Pantheon Books, 1978.

—. Power/Knowledge: Selected interviews and other writings 1972-1977. New York: Pantheon Books, 1980.

-. Society must be defended. Lectures at the Collège de France 1975-76. New Yotk: Picador, 2003.

Goffman, Erving. Estigma: la identidad deteriorada. Buenos Aires: Amorrortu, 2012.

Herek, Gregory M. «Beyond Homophobia: Thinking About Sexual Stigma and Prejudice in the Twenty-First Century». Sexuality Research and Social Policy 1.2 (2004): 6-24.

Jones, Christopher P. «Stigma: tattooing and branding in Graeco-Roman antiquity». The Journal of Roman Studies 77 (1987): 139-155.

Juliano, Dolores. Tomar la palabra. Mujeres, discursos y silencios. Barcelona: Ediciones Bellaterra, 2017.

Latimer, Joanna. «Repelling neoliberal world-making? How the ageing-dementia relation is reassembling the social». The Sociological Review Monographs 66.4 (2018): 832-856.

Link, Bruce y Jo Phelan. «Conceptualizing stigma». Annual Review of Sociology 27 (2001): 363-385.

Major, Brenda y Laurie T. O'Brien. «The social psychology of stigma». Annual Review of Psychology 56 (2005): 393-421.

Martínez-Palacios, Jone. «Exclusión, profundización democrática e interseccionalidad». Investigaciones Feministas 8.1 (2017): 53-71.

Panchakis, John E., Mark L. Hatzenbuehler, Katie Wang, Charles L. Burton, Forrest W. Crawford, Jo C. Phelan y Bruce G. Link. «The burden of stigma on health and well-being: a taxonomy of concealment, course, disruptiveness, aesthetics, origin and peril across 93 stigmas». Personality and Social Psychology Bulletin 44.4 (2018): 451-474.

Parker, Richard y Peter Aggleton. «HIV and AIDS-related stigma and discrimination: a conceptual framework and implications for action». Social Science and Medicine 57.1 (2003): 13-24.

Pescosolido, Bernice A. y Jack K. Martin. «The stigma complex». Annual Review of Sociology 41 (2015): 87-116. 
Phelan, Jo C., Bruce G. Link y John F. Dovidio. «Stigma and prejudice: One animal or two?». Social Science and Medicine 67 (2008): 358-367.

Severs, Eline, Karen Celis y Silvia Erzeel. «Poder, privilegio y desventaja: teoría interseccional y representación política». Revista de Investigaciones Feministas 8.1 (2017): 41-51.

Stangor, Charles y Christian S. Crandall. «Threat and the social construction of stigma». The Social Psychology of Stigma. Eds. Todd F. Heatherton, Robert E. Kleck, Michelle R. Hebl y Jay G. Hull. New York, 2000. 62-88.

Tyler, Imogen. «Resituating Erving Goffman: from stigma power to black power». The Sociological Review Monographs 66.4 (2018): 744-765.

-. «The hyeroglyphics of the border: racial stigma in neoliberal Europe». Ethnic and Racial Studies 41.10 (2018a): 1783-1801.

-. Revolting subjects. Social abjection and resistance in neoliberal Britain. London: Zed Books, 2013.

Tyler, Imogen y Tom Slater. «Rethinking the sociology of stigma». The Sociological Review Monographs 66.4 (2018): 721-743.

Wacquant, Loic. «Territorial stigmatization in the age of advanced marginality». Thesis Eleven 91 (2007): 66-77.

Women's Link Worldwide. Madres en las redes de trata. Derechos robados. Informe 8, 2017.

Young, Iris Marion. La justicia y la política de la diferencia. Madrid: Ediciones Cátedra, 2000. 